\title{
Enhancing the security of Cloud computing via unscented Kalman filter; with statistical analysis about power consumption
}

\author{
Mohamadreza Mohamadzadeh \\ ${ }^{1}$ Department of Electronic Engineering, Khorasan Razavi,Neyshabur,Science and Research branch,Islamic Azad University, \\ Neyshabur,Iran \\ mohamadreza_mohamadzadeh@hotmail.com \\ m_mohamadzadeh.talent@yahoo.com
}

Received: June 22, 2019. Revised: July 5, 2021. Accepted: October 10, 2021. Published: November $26,2021$.

\begin{abstract}
To migrate to cloud computing or other new generation of computational and communicational network we should making some laws about limitations on such networks. We should clarify clearly the level of accessions to resources and/or the ability to sending or receiving files through such network. We can avoid unpredicted and undesired works by using of law makings. Furthermore, besides of law making we can use evolutionary and/or intelligence techniques for estimation and prediction about parameters that deal with controlling of our network - for example in this paper we introduce one type of such intelligence algorithms which named as unscented Kalman filter. By giving some raw inputs about treatments and resources of such networks we're able to estimate and predict about lots of different conditions of these networks. For example, we should have an estimation and prediction algorithm to be able to track and trace the hackers or crackers, if they're permeating to our network. Or we should estimate the amount of users that will use particular software or application on a specific hour through such network. Also, if we study users treatment on a specific network, like cloud computing, we're able to estimate and predict their keen and eagerness about new released software or application; because if we know how people treat and face and react with new emerging software we're able to construct and program our applications more precisely. In this paper, at first we tell about cloud computing - we define it completely and discuss about all aspects of this new generation of internet - also in the first section we discuss about efficiency of cloud computing in saving of energy and tell differences between the amount of energy that cloud resources use and the amount of energy that other computerized frames were use. We proof our suggestions and ideas by the means of analytical and mathematical analysis; after that when we understand about all of its concepts, a brief review about Microsoft decisions on cloud computing were present, we review new systems and software's of Microsoft that equipped with cloud computing and study the advantages and disadvantages of these new services which are equipped with cloud computing. In the next section by the knowing of whole concepts and key features of this network and also by knowing the critical uses of this network on different industries we introduce fixedinterval smoothers; which can be used for estimation
\end{abstract}

and prediction of different parameters in such network. For example, we can use this for estimation and prediction about permeating of hackers or for predicting the amount of users that will use special part of our network in a particular period of time or by tracking and tracing the packets or even users. By performing such activities we're able to eliminate malicious and spyware treatments from the beginning points of our network. Also by the means of mathematical analysis in this paper, we demonstrate all of our suggestions and according to these proofs we conclude about workability and liability of such network.

Keywords- Computer generations, Cloud platforms, evolutionary algorithms, Kalman filtering, fixed-lag smoother.

\section{INTRODUCTION}

Cloud computing is a pay-per-use model for enabling available, convenient, on-demand network access to a shared pool of configurable computing resources (e.g., networks, servers, storage, applications, services) that can be rapidly provisioned and released with minimal management effort or service provider interaction. This cloud model promotes availability and is comprised of five key characteristics, three delivery models, and four deployment models.

On-demand self-service: A consumer can unilaterally provision computing capabilities, such as server time and network storage, as needed without requiring human interaction with each service's provider.

Ubiquitous network access: Capabilities are available over the network and accessed through standard mechanisms that promote use by heterogeneous thin or thick client platforms (e.g., mobile phones, laptops, and PDAs). 
Location independent resource pooling: The provider's computing resources are pooled to serve all consumers using a multi-tenant model, with different physical and virtual resources dynamically assigned and reassigned according to consumer demand. The customer generally has no control or knowledge over the exact location of the provided resources. Examples of resources include storage, processing, memory, network bandwidth, and virtual machines.

Rapid elasticity: Capabilities can be rapidly and elastically provisioned to quickly scale up and rapidly released to quickly scale down. To the consumer, the capabilities available for rent often appear to be infinite and can be purchased in any quantity at any time.

Pay per use: Capabilities are charged using a metered, fee-for-service, or advertising based billing model to promote optimization of resource use. Examples are measuring the storage, bandwidth, and computing resources consumed and charging for the number of active user accounts per month. Clouds within an organization accrue cost between business units and may or may not use actual currency.

The dematerialization strategy for altering the IT supply chain with thin-clients and computing resources in the cloud can return both environmental and economic savings. The suggestion in literature [Williams 2004, Byung-Chul et. al 2006] that increased PC reuse rates and upgradability potential will lead to environmental savings is correct; however no trends in this area are emerging. The usage of thinclients and cloud computing may turn out to be the preferable route to reduce the environmental impacts of computing. The issue of computer obsolescence may become less of an issue because the thin-client's performance can increase with additional computational resources from remote servers. This effectively extends the life of the equipment used in office computing as computational services in the cloud can always be upgraded. C omputing as a service is an exciting proposition and will potentially grow as internet bandwidth becomes cheaper, faster, and more reliable. The switch to the cloud also has the ability to promote innovation and entrepreneurship because small businesses and start-ups will face much lower capital costs for IT and computing. A large focus with computing revolves around the energyefficiency of chips, however the cloud provides a path for dematerialization that does not rely on re-use and upgradability.

Cloud software takes full advantage of the cloud paradigm by being service oriented with a focus on statelessness, low coupling, modularity, and semantic interoperability.

\section{Greenhouse Gas Equivalent for Production and Use Phases for Computers}

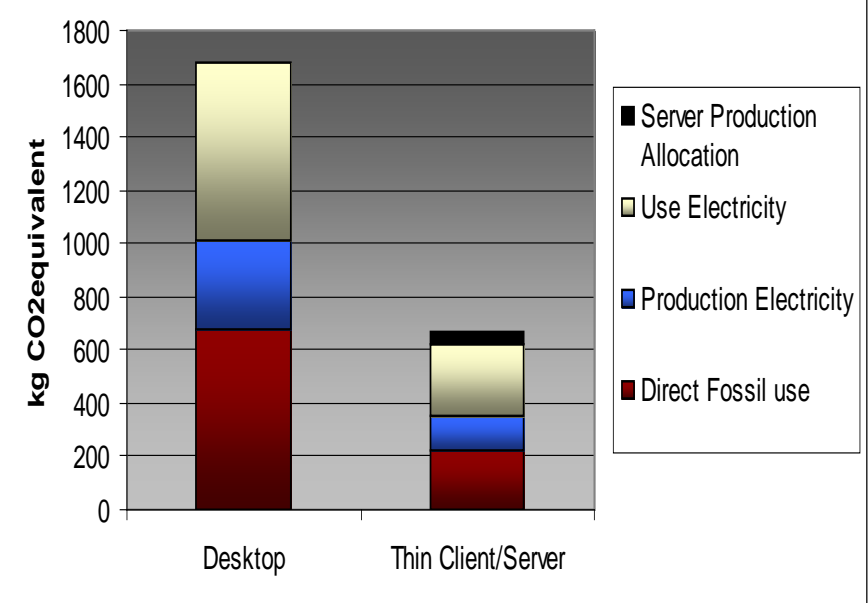

Cloud Software as a Service (SaaS), the capability provided to the consumer is to use the provider's applications running on a cloud infrastructure and accessible from various client devices through a thin client interface such as a Web browser (e.g., web-

\section{Total Impact for Small Business with 100 Machines}

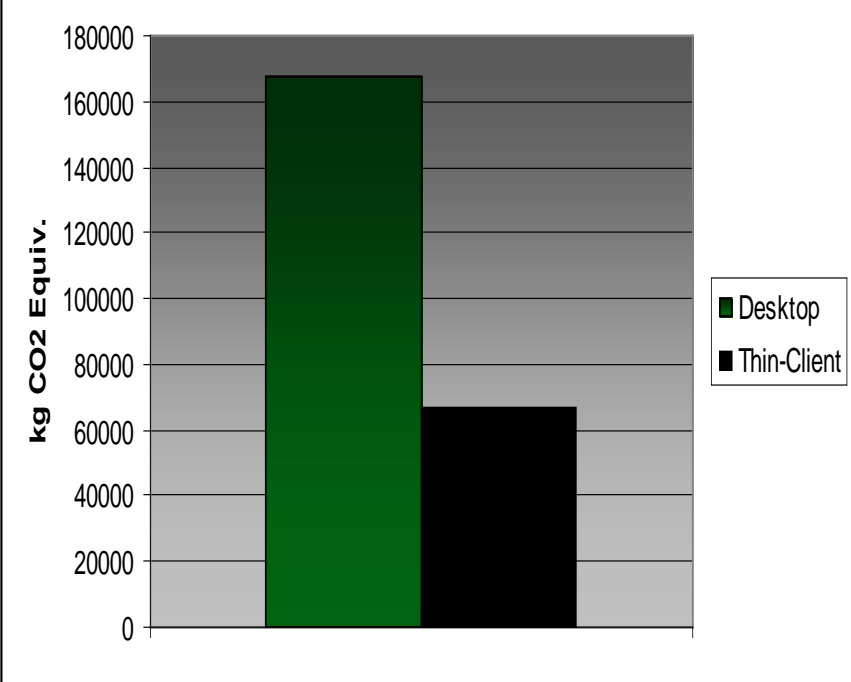

based email). The consumer does not manage or control the underlying cloud infrastructure, network, servers, operating systems, storage, or even individual application capabilities, with the possible exception of limited user-specific application configuration settings. Cloud Platform as a Service (PaaS), the capability provided to the consumer is to deploy onto the cloud infrastructure consumer-created applications using 
programming languages and tools supported by the provider (e.g., java, python, .Net). The consumer does not manage or control the underlying cloud infrastructure, network, servers, operating systems, or storage, but the consumer has control over the deployed applications and possibly application hosting environment configurations.

Cloud Infrastructure as a Service (IaaS), the capability provided to the consumer is to rent processing, storage, networks, and other fundamental computing resources where the consumer is able to deploy and run arbitrary software, which can include operating systems and applications. The consumer does not manage or control the underlying cloud infrastructure but has control over operating systems, storage, deployed applications, and possibly select networking components (e.g., firewalls, load balancers).

The server can handle the computational power of approximately $20 \mathrm{~m}$ achines performing common business tasks and applications (Linux Terminal Service Project 2009). The cost of the server is allocated at a ratio of $1 / 20$.

$$
\text { CostAllocation }=\frac{\text { ServerCost }}{\text { ThinClients }}=\frac{\$ 4000}{20}=\$ 150_{\text {per_workstation }}
$$

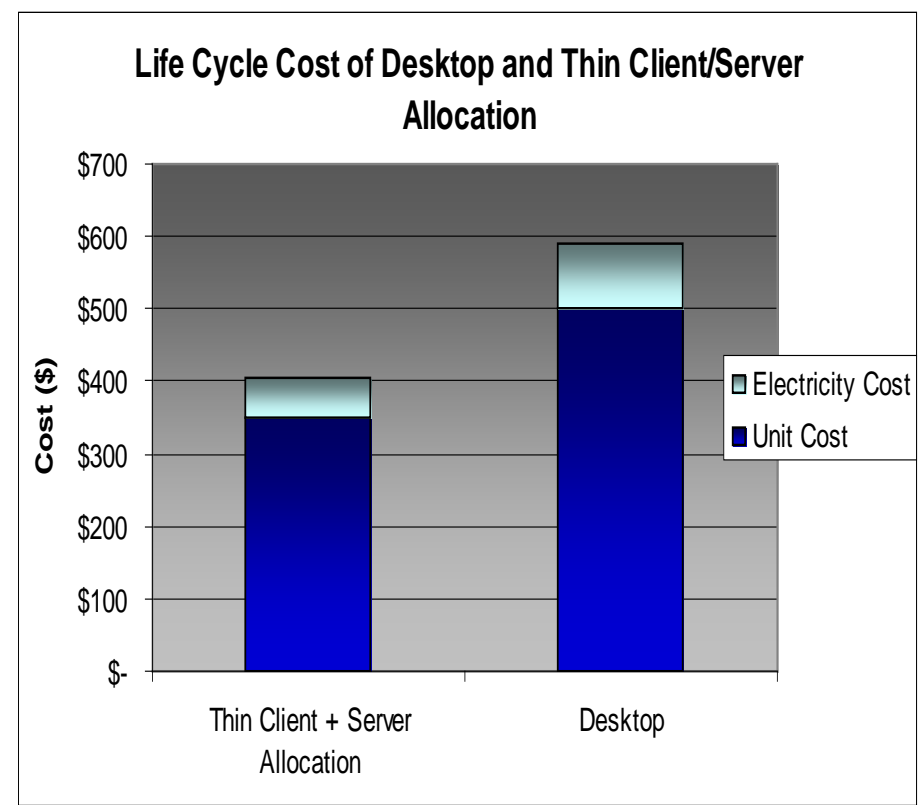

If the computational power and data storage services were provided by an external agent even greater efficiency gains would be obtained through increased optimization and economies of scale for cooling. In addition, a small company would need less internal information technology staff to run their systems if the servers were offsite.

The utilization rate for the server's computational power would increase because the cloud service company can take advantage of national economies of scale. The peak computational demand required by businesses would be more evenly distributed because of the time zones in the country. A three year time span was used for this analysis, but there are no technical constraints limiting the thin-client to such a short-lifespan. It is feasible that the hardware could last much longer (5+ years) because they are not as performance-dependent as a d esktop machine. The servers can be upgraded centrally rather than the individual desktop machines, but with the thin-client setup, users still receive the same unit of computing at their desks.

If the internet can reliably and quickly route business machine computational power, the market for traditional desktop machines could be affected. Companies that provide desktop business machines (Dell) would need to shift their business models. A greater reliance on high-performance centralized servers will likely drive the market for computing in coming years. Innovation in computational service contracts will emerge and small companies will have less risk investing in IT equipment. Cloud providers could provide the thin-clients for free or a monthly rental rate similar to the approach currently utilized by cable companies. Because the thin-clients do not need to be upgraded as frequently because computation occurs offsite, cloud providers could re-use thinclients further decreasing the environmental burdens.

With any reliance on new technology, businesses may be hesitant to fully move to a cloud based thin-client service. I f all computational and data storage is provided live in the cloud, a business is completely reliant on their internet connection. If for any reason the internet goes down, all the workstations would be effectively useless and productivity would suffer.

Businesses may also be wary of outsourcing their sensitive data to external third party companies that provide a cloud service. Privacy issues could hinder deployment of full cloud computing as the importance of data security can trump potential economic and environmental gains.

If computation and data storage is provided as a service, there could be considerable business costs required to switch service providers. B usinesses would essentially be locked into their service provider and even if another company could offer cheaper rates, a considerable transaction cost would have to be considered.

The only indicator used in this analysis is global warming potential. $\mathrm{T}$ here are other environmental impacts in computer manufacturing such as human and aquatic toxicity that are important to consider. A s much as $70 \%$ of the human toxicity impact occurs during the disposal stage of the extended supply chain [Byung-Chul et. al 2004]. H owever, because the switch to thin-clients (and the cloud) is essentially a 
dematerialization strategy for pollution prevention, all of these other environmental impacts associated with component manufacturing will be reduced.

When data storage and computation is provided as a service that is routed through the internet, additional network components and electricity will be required. This study omits the impacts associated with an increasing demand requirement for internet infrastructure.

The raw values for energy requirements for PC manufacturing published by Williams 2004 a lready have embedded uncertainty and then they are scaled again in this study to account for thin-client manufacturing impacts. There is a considerable error margin with our projected GWP reduction of $\% 60$.

\section{EVALUATING THE REVOLUTION THAT}

\section{MADE BY CLOUD COMPUTING ON DIFFERENT}

\section{INDUSTRIES}

In recent years, with the cloud computing technology Introduced into China, the experts and scholars in the various fields have done a lot of researches for cloud computing infrastructure construction, efficient use and share. At first, the article analyzed the feasibility of building regional digital instruction resources that based on cloud computing technology, and specifically discussed about cloud computing technology to build the two main constructions of the regional universities of digital instruction resources: cloud services platform and university digital instruction resources. It also proposed that building two main constructions is the core, improving management is the guarantee, share is the purpose and evaluation is a follow-up to improve. Through the integration of high-quality instruction resources in the region promoted the centralized management and a balanced distribution to improve the overall quality of instruction in the universities within the region.

As computers and the Internet play an increasingly important part in people's lives - at work, at school, and at home-making technology accessible to people with a w ide range of abilities is an imperative. Accessible technology makes it easier for people with visual, dexterity, cognitive and speech needs to use computers and other devices to communicate and share information, access government services, and secure and retain employment.

According to the United Nations Convention on Persons with Disabilities, disability affects between 15 percent and 20 pe rcent of the population in every country worldwide, and the incidence of disability in industrialized nations is increasing as their populations get older. A Forrester Research Inc. study commissioned by Microsoft found that 57 percent of working-age computer users (18-64 years old) would likely benefit from accessible technology because of difficulties and impairments that affect their computer use. As the population continues to age, the number of computer users in the 65-74 age range will increase significantly, as will the number of people who would benefit from accessible technology.

For more than 25 years, Microsoft has been at the forefront of efforts to make computers and the Internet more accessible. These efforts include:

- Building accessibility options into Windows and other Microsoft products that let users personalize the computer display, mouse, keyboard, sound, and speech options.

- Working with AT companies to facilitate the creation of software and hardware products such as screen readers, magnifiers, and specialty keyboards that enable individuals with significant vision, hearing, dexterity, language or learning needs to use computers.

- Creating developer technologies and tools that make it easier to build AT products and accessible applications that interoperate and work well together.

Today, a vibrant worldwide ecosystem of accessible technology tools, technologies, and solutions is helping enable and empower millions of people of all ages and abilities. However, as the technology landscape continues to evolve, so too must the building blocks that support this accessible technology ecosystem.

Of particular significance is the rise of "cloud computing," which encompasses a major shift in how technology will be used in the years ahead and what people and organizations can do with it. In the simplest terms, cloud computing represents the convergence of smart Internet-connected devices, innovative software applications, and data storage and services hosted on the Internet. This convergence of hosted data storage and services, applications, and devices is sometimes referred to as "three screens and a cloud." (The three screens being laptop or desktop computers, smartphones, and TVs.)

Advances in "client-based" operating systems like Windows 7 and applications like Microsoft Office 2010 are powering many of these devices with more intuitive user interfaces and the ability to perform increasingly complex computing functions, such as data visualization and real-time simulation of $3 \mathrm{D}$ environments. At the same time, development of large data centers capable of storing huge volumes of data and hosting a rapidly expanding universe of online applications and services is opening new realms of communication, collaboration, commerce, learning, and entertainment. Microsoft Office 365, the cloudbased version of Microsoft Office, utilizes the power 
of these online capabilities to simplify sharing, collaboration and storage of Office documents, spreadsheets and presentations. Microsoft also offers many other online services, solutions, and platforms that are enabling individuals, businesses, and governments to take advantage of the convenience and flexibility of the cloud. For individuals, the cloud has the potential to offer seamless, on-demand access to content, social networks, and online services across a range of devices. For organizations, the cloud offers the ability to dynamically scale IT capacity depending on evolving needs, better manage spending on IT infrastructure, and concentrate more resources on core business functions.

Just as the cloud itself is a work in progress, so, too, are Microsoft's efforts to enable the cloud for people of all abilities. As we continue to invest in building a more seamless cloud-based spectrum of solutions, we will also back the contributions of other developers of accessible products, services, and content. We believe that a healthy, market-driven ecosystem comprised of many products, business models, and development philosophies is the fastest route to achieving a more accessible cloud.

Many different approaches are represented in the current ecosystem, and Microsoft supports many of them. For example, Microsoft is working in partnership with an organization called LucyTech and with government organizations worldwide to pilot a commercially-viable model for cloud-based accessibility solutions, which will be made available at little or no cost to consumers. Although LucyTech will build on existing cloud, platform, and application investments, it is pushing the boundaries of existing technologies and cloud infrastructure and will include some new cloud-based service offerings to fill critical gaps. It is a distributed accessibility solution with the promise to provide globally available AT solutions.

Another international effort we support is the Global Public Inclusive Infrastructure (GPII). The goal of GPII is to increase awareness of the existing accessibility infrastructure (such as APIs, operating system features, and tools) and accelerate development of new accessibility "building blocks" that make the benefits of the cloud more easily available to everyone. Participants in the GPII process agree that a client device and associated operating system form the foundation of accessibility solutions and are the first line of functionality that people will use. There also is a shared understanding that cloud-based services offer significant opportunities to complement and extend those built-in capabilities to create new and better customer-ready features. For example, a fullydeveloped accessible cloud infrastructure would enable people who cannot use standard interfaces and content to use a broadband connection to invoke the interface adoptions they need, automatically, on any device, anywhere and anytime. The GPII vision aligns with Microsoft's long-standing desire for an adaptive computing environment. In some respects, GPII is helping clarify the vision and need for better cloudconnected accessibility products and services, while the LucyTech project is focusing on the development and delivery of a working solution that fully utilizes existing Microsoft cloud technologies, services, and commercial AT products to realize this vision. Microsoft sees value in both projects, so is participating in and supporting both efforts.

Governments around the world are increasingly aware of the enormous potential of cloud computing to benefit citizens and society. Microsoft has developed a set of policy principles to support its commitment to both digital inclusion and to the cloud. Microsoft believes these principles are at the core of effective policy frameworks for accessibility and the cloud, and will encourage the widespread development and implementation of accessible technologies, products, programs, and services. These policies include:

\section{Harmonizing International Standards and} Procurement Approaches: Market-led, globally harmonized standards and the procurement policies that reference them are the foundation of accessible and interoperable IT products and cloud services. Globally-harmonized standards bring major advantages to both business and individual users of accessible technology. Because the speed of innovation in the IT sector can outpace legislative measures put in place to encourage accessibility and promote cloud services, Microsoft believes it is important that globally harmonized standards constitute the basis of product development.

Promoting Effective Conformance: Accessible technology can be advanced most effectively by minimizing undue regulatory burden. When standards for cloud and accessibility are referenced in government procurement policies, Microsoft supports effective and rigorous conformance mechanisms that provide adequate assurance of compliance, encourage innovation, and promote consumer choice. One such example is the voluntary supplier declaration of accessibility, as defined in the ISO/IEC 17050 standard and used in a number of countries around the world.

Promoting Interoperability: Interoperability is crucial to creating an accessible cloud computing environment for users with a broad range of abilities. It is a key feature of Microsoft accessible technologies and we advocate for public policies that allow a variety of complementary ways to achieve it. We believe that governments should not engineer 
interoperability by prescribing specific approaches and excluding others, but instead should allow IT vendors and purchasers to use the solutions that best suit their needs. AT/IT interoperability standards such as I SO 13066 will help ensure that vendors' cloud-ready technologies are also interoperable with assistive technology. Aligning user needs with a dynamic and growing array of devices, technology platforms, development tools, assistive technologies, and services is a complex challenge that will require industry-wide innovation and collaboration, appropriate government policies, and the active involvement of the disability community.

Ensuring Technology Neutrality: Technologyneutral policies promote innovation, eliminate barriers to trade and market access, enhance competition, and prevent bias in government procurement. Microsoft believes that a flexible cloud policy approach based on a commitment to technology neutrality and choice is particularly appropriate in the rapidly evolving IT environment. Customers and governments today increasingly rely on a combination of proprietary and open source software, as well as on open standards and proprietary standards, to develop accessibility solutions. By encouraging choice, governments will enable the broadest possible array of solutions for people with disabilities and drive down the cost of building accessible mainstream products.

Enhancing the Ecosystem: In order for technology companies to invest in and develop accessible cloud solutions, they must understand user needs and be confident that there will be a market for such solutions. Today, the accessible technology PC ecosystem is a vibrant and healthy collection of more than 350 products that offer tremendous customer benefit. Assistive technologies for the cloud will require similar breadth and depth. Microsoft believes that governments should take steps to ensure accessibility is not undermined by cloud policies that put the existing AT ecosystem at risk or that diminish the ability of entrepreneurs to continue serving the disability community.

\section{UNSCENTED KALMAN FILTER:}

When the state transition and observation models that is, predict and update functions $f$ and $h$ - are highly non-linear, the extended Kalman filter can give particularly poor performance. This is because the covariance is propagated through linearization of the underlying non-linear model. The unscented Kalman filter (UKF) uses a deterministic sampling technique known as the unscented transform to pick a minimal set of sample points (called sigma points) around the mean. These sigma points are then propagated through the non-linear functions, from which the mean and covariance of the estimate are then recovered. The result is a filter which more accurately captures the true mean and covariance. (This can be verified using Monte Carlo sampling or through a Taylor series expansion of the posterior statistics.) In addition, this technique removes the requirement to explicitly calculate Jacobeans, which for complex functions can be a difficult task in itself (i.e., requiring complicated derivatives if done analytically or being computationally costly if done numerically).

\section{Predict:}

As with the EKF, the UKF prediction can be used independently from the UKF update, in combination with a linear (or indeed EKF) update, or vice versa.

The estimated state and covariance are augmented with the mean and covariance of the process noise.

$\mathbf{x}_{k-1 \mid k-1}^{a}=\left[\begin{array}{ll}\hat{\mathbf{x}}_{k-1 \mid k-1}^{T} & E\left[\mathbf{w}_{k}^{T}\right]\end{array}\right]^{T}$
$\mathbf{P}_{k-1 \mid k-1}^{a}=\left[\begin{array}{cc}\mathbf{P}_{k-1 \mid k-1} & 0 \\ 0 & \mathbf{Q}_{k}\end{array}\right]$

A set of $2 \mathrm{~L}+1$ sigma points is derived from the augmented state and covariance where $\mathrm{L}$ is the dimension of the augmented state.

$\chi_{k-1 \mid k-1}^{0}=\mathbf{x}_{k-1 \mid k-1}^{a}$

$\chi_{k-1 \mid k-1}^{i}=\mathbf{x}_{k-1 \mid k-1}^{a}+\left(\sqrt{(L+\lambda) \mathbf{P}_{k-1 \mid k-1}^{a}}\right)_{i}$

$i=1 . . L$

$\chi_{k-1 \mid k-1}^{i}=\mathbf{x}_{k-1 \mid k-1}^{a}-\left(\sqrt{(L+\lambda) \mathbf{P}_{k-1 \mid k-1}^{a}}\right)_{i-L}$

$i=L+1, \ldots 2 L$

Where:

$\left(\sqrt{(L+\lambda) \mathbf{P}_{k-1 \mid k-1}^{a}}\right)_{i}$

is the $\mathrm{i}$-th column of the matrix square root of:

$(L+\lambda) \mathbf{P}_{k-1 \mid k-1}^{a}$

Using the definition: square root $\mathrm{A}$ of matrix $\mathrm{B}$ satisfies:

$B \triangleq A A^{T}$.

The matrix square root should be calculated using numerically efficient and stable methods such as the Cholesky decomposition. 
The sigma points are propagated through the transition function $\mathrm{f}$.

$\chi_{k \mid k-1}^{i}=f\left(\chi_{k-1 \mid k-1}^{i}\right) \quad i=0 . .2 L$

Where $f: R^{L} \rightarrow R^{|\mathbf{X}|}$. The weighted sigma points are recombined to produce the predicted state and covariance.

$\hat{\mathbf{x}}_{k \mid k-1}=\sum_{i=0}^{2 L} W_{s}^{i} \chi_{k \mid k-1}^{i}$

$\mathbf{P}_{k \mid k-1}=\sum_{i=0}^{2 L} W_{c}^{i}\left[\chi_{k \mid k-1}^{i}-\hat{\mathbf{x}}_{k \mid k-1}\right]\left[\chi_{k \mid k-1}^{i}-\hat{\mathbf{x}}_{k \mid k-1}\right]^{T}$

Where the weights for the state and covariance are given by:

$$
\begin{aligned}
& W_{s}^{0}=\frac{\lambda}{L+\lambda} \\
& W_{c}^{0}=\frac{\lambda}{L+\lambda}+\left(1-\alpha^{2}+\beta\right) \\
& W_{s}^{i}=W_{c}^{i}=\frac{1}{2(L+\lambda)} \\
& \lambda=\alpha^{2}(L+\kappa)-L
\end{aligned}
$$

$\alpha$ and $\kappa$ control the spread of the sigma points. $\beta$ is related to the distribution of $x$. Normal values are $\alpha=10^{-3}, \kappa=0$ and $\beta=2$. If the true distribution of $x$ is Gaussian, $\beta=2$ is optimal.

\section{Update:}

The predicted state and covariance are augmented as before, except now with the mean and covariance of the measurement noise.

$\mathbf{x}_{k \mid k-1}^{a}=\left[\begin{array}{ll}\hat{\mathbf{x}}_{k \mid k-1}^{T} & E\left[\mathbf{v}_{k}^{T}\right]\end{array}\right]^{T}$

$\mathbf{P}_{k \mid k-1}^{a}=\left[\begin{array}{cc}\mathbf{P}_{k \mid k-1} & 0 \\ 0 & \mathbf{R}_{k}\end{array}\right]$

As before, a set of $2 \mathrm{~L}+1$ sigma points is derived from the augmented state and covariance where $\mathrm{L}$ is the dimension of the augmented state.

$\chi_{k \mid k-1}^{0}=\mathbf{x}_{k \mid k-1}^{a}$

$\chi_{k \mid k-1}^{i}=\mathbf{x}_{k \mid k-1}^{a}+\left(\sqrt{(L+\lambda) \mathbf{P}_{k \mid k-1}^{a}}\right)_{i}$

$i=1$..L

$$
\begin{aligned}
& \chi_{k \mid k-1}^{i}=\mathbf{x}_{k \mid k-1}^{a}-\left(\sqrt{(L+\lambda) \mathbf{P}_{k \mid k-1}^{a}}\right)_{i-L} \\
& i=L+1, \ldots 2 L
\end{aligned}
$$

Alternatively if the UKF prediction has been used the sigma points themselves can be augmented along the following lines:

$\underset{\text { Where: }}{\chi_{k \mid k-1}:=\left[\begin{array}{ll}\chi_{k \mid k-1}^{T} & E\left[\mathbf{v}_{k}^{T}\right]\end{array}\right]^{T} \pm \sqrt{(L+\lambda) \mathbf{R}_{k}^{a}}}$

$\mathbf{R}_{k}^{a}=\left[\begin{array}{cc}0 & 0 \\ 0 & \mathbf{R}_{k}\end{array}\right]$

The sigma points are projected through the observation function $h$.

$\gamma_{k}^{i}=h\left(\chi_{k \mid k-1}^{i}\right) \quad i=0 . .2 L$

The weighted sigma points are recombined to produce the predicted measurement and predicted measurement covariance.

$\hat{\mathbf{z}}_{k}=\sum_{i=0}^{2 L} W_{s}^{i} \gamma_{k}^{i}$

$\mathbf{P}_{z_{k} \tau_{k}}=\sum_{i=0}^{2 L} W_{c}^{i}\left[\gamma_{k}^{i}-\hat{\mathbf{z}}_{k}\right]\left[\gamma_{k}^{i}-\hat{\mathbf{z}}_{k}\right]^{T}$

The state-measurement cross-covariance matrix,

$\mathbf{P}_{x_{k} \approx k}=\sum_{i=0}^{2 L} W_{c}^{i}\left[\chi_{k \mid k-1}^{i}-\hat{\mathbf{x}}_{k \mid k-1}\right]\left[\gamma_{k}^{i}-\hat{\mathbf{z}}_{k}\right]^{T}$ is used to compute the UKF Kalman gain.

$$
K_{k}=\mathbf{P}_{x_{k} z_{k}} \mathbf{P}_{z_{k} z_{k}}^{-1}
$$

As with the Kalman filter, the updated state is the predicted state plus the innovation weighted by the Kalman gain,

$$
\hat{\mathbf{x}}_{k \mid k}=\hat{\mathbf{x}}_{k \mid k-1}+K_{k}\left(\mathbf{z}_{k}-\hat{\mathbf{z}}_{k}\right)
$$

And the updated covariance is the predicted covariance, minus the predicted measurement covariance, weighted by the Kalman gain.

$$
\mathbf{P}_{k \mid k}=\mathbf{P}_{k \mid k-1}-K_{k} \mathbf{P}_{z_{k} z_{k}} K_{k}^{T}
$$




\section{CONCLUSION}

The purpose and idea of this paper can divide into three sections. In the first section of this paper you can study about cloud computing and its influences on different computerized systems and networks. In this section, principles of cloud computing, were explain in brief and all of the required information for attaining comprehensive comprehend about cloud computing were present. The second section of this paper were allocate into programs of the biggest computer and software corporation in the world which is known as Microsoft Corp. In this section authors provide complete and comprehensive details about future programs and estimations of Microsoft about prospect of this technology. After studying this section, the first question that the reader may have is: Security and reliability of this network. Authors of this paper answers these kinds of questions in section three, where a new algorithm were implemented on cloud platforms for attaining better security.

\section{REFERENCES:}

[1] mohamadreza mohamadzadeh " An overview of the effects of processing on C loud Computing dramatic present and provide new security solutions "; published by the life science journal - marsland press, 2013; USA

[2] http://www.cs.unc.edu

[3] http://info.acm.org/pubs/toc/CRnotice.html

[4] Microsoft's Accessible Technology Vision and Strategy; September 2011.

[5] www.wikipedia.org.

[6] C.G. Atkeson and J.M. Hollerbach. 1985. "Kinematic features

of unrestrained vertical arm movements," Journal of Neuroscience, 5:2318-2330.

Ali Azarbayejani and Alex Pentland. June 1995. "Recursive Estimation

of Motion, Structure, and Focal Length," IEEE Trans. Pattern

Analysis and Machine Intelligence, June 1995, 17(6).

[7] Ronald Azuma and Mark Ward. 1991. "SpaceResection by

Collinearity: Mathematics Behind the Optical Ceiling Head-Tracker,"

UNC Chapel Hill Department of Computer Science technical report TR 91-048 (November 1991).

[8] Ronald Azuma and Gary Bishop. 1994. "Improving Static and

Dynamic Registration in an Optical See-Through HMD," SIGGRAPH

Conference Proceedings, Annual Conference Series, pp. 197-204 ACM SIGGRAPH, Addison Wesley, July 1994. ISBN0-201-60795-6.

[9] Ronald Azuma. 1995. "Predictive Tracking for Augmented Reality,"

Ph.D. dissertation, University of North Carolina at Chapel

Hill, TR95-007.

[10] Ted J. Broida and Rama Chellappa. 1986. "Estimation of object

motion parameters from noisy images," IEEE Trans. Pattern Analysis

and Machine Intelligence, January 1986, 8(1), pp. 90-99.

[11] R. G. Brown and P. Y. C. Hwang. 1992. Introduction to Random

Signals and Applied Kalman Filtering, 2nd Edition, John Wiley \&

Sons, Inc.

\section{Creative Commons Attribution License 4.0 (Attribution 4.0 International, CC BY 4.0)}

This article is published under the terms of the Creative Commons Attribution License 4.0 https://creativecommons.org/licenses/by/4.0/deed.en US 